\title{
Presentation outside office hours does not negatively influence treatment times for reperfusion therapy for acute ischemic stroke
}

\author{
A. E. Groot ${ }^{1}$ - H. de Bruin ${ }^{1}$-T. T. M. Nguyen ${ }^{2} \cdot$ M. Kappelhof ${ }^{3} \cdot$ F. de Beer ${ }^{4} \cdot$ M. C. Visser ${ }^{5}$. C. P. Zwetsloot ${ }^{6}$. \\ P. H. A. Halkes ${ }^{7}$. J. de Kruijk ${ }^{8}$. W. D. M. van der Meulen ${ }^{9}$. T. C. van der Ree ${ }^{10}$ - V. I. H. Kwa ${ }^{11}$. S. M. van Schaik ${ }^{12}$. \\ L. Hani ${ }^{13}$ - R. van den Berg ${ }^{3}$. M. E. S. Sprengers ${ }^{3}$. S. D. Roosendaal ${ }^{3}$. B. J. Emmer ${ }^{3}$. P. J. Nederkoorn ${ }^{1}$. \\ C. B. L. M. Majoie ${ }^{3}$. Y. B. W. E. M. Roos ${ }^{1}$. J. M. Coutinho ${ }^{1}$ (1)
}

Received: 16 April 2020 / Revised: 19 July 2020 / Accepted: 21 July 2020 / Published online: 31 July 2020

(c) The Author(s) 2020

\begin{abstract}
Background Treatment outside office hours has been associated with increased workflow times for intravenous thrombolysis (IVT) in acute ischemic stroke (AIS). Limited data suggest that this "off-hours effect" also exists for endovascular treatment (EVT). We investigated this phenomenon in a well-organized acute stroke care region in the Netherlands.

Methods Retrospective, observational cohort study of consecutive patients with AIS who received reperfusion therapy in the Greater Amsterdam Area, consisting of 14 primary stroke centers and 1 comprehensive stroke center (IVT: 2009-2015, EVT: 2014-2017). Office hours were defined as presentation during weekdays between 8 AM and 5 PM, excluding National Festive days. Primary outcome was door-to-treatment time (door-to-needle [DNT] for IVT, door-to-groin [DGT] for EVT). For DGT, we used the door time of the first hospital. Other outcomes were in-hospital mortality, modified Rankin Scale (mRS) score at 90 days and symptomatic intracranial hemorrhage (sICH). We performed multivariable linear and logistic regression analyses and used multiple imputation to account for missing values.

Results In total, 59\% (2450/4161) and 61\% (239/395) of patients treated with IVT and EVT, respectively, presented outside office hours. Median DNT was minimally longer outside office hours (32 vs. $30 \mathrm{~min}, p=0.024$, adjusted difference $2.5 \mathrm{~min}$, 95\% CI 0.7-4.2). Presentation outside office hours was not associated with a longer DGT (median 130 min for both groups, adjusted difference $7.0 \mathrm{~min}, 95 \% \mathrm{CI}-4.2$ to 18.1 ). Clinical outcome and sICH rate also did not differ.

Conclusion Presentation outside office hours did not lead to clinically relevant treatment delays for reperfusion therapy in patients with AIS.
\end{abstract}

Keywords Acute ischemic stroke $\cdot$ Off-hour presentation $\cdot$ Functional outcome $\cdot$ Treatment times $\cdot$ Workflow

\section{Introduction}

The beneficial effect of intravenous thrombolysis (IVT) and endovascular treatment (EVT) in patients with acute ischemic stroke (AIS) is highly time dependent and a reduction in treatment times increases the chance of good clinical outcome [1,2]. Stroke patients often present outside of office hours, which may cause treatment delay, for example due to

Electronic supplementary material The online version of this article (https://doi.org/10.1007/s00415-020-10106-z) contains supplementary material, which is available to authorized users.

J. M. Coutinho

j.coutinho@amsterdamumc.nl

Extended author information available on the last page of the article lower staff attendance, and decreased availability of imaging [3]. Previous studies on the existence of such an "offhours effect" have yielded conflicting results, observing both longer and shorter treatment times for patients presenting outside office hours [4-7]. Moreover, these studies mostly focused on door-to-needle times (DNT) for IVT, and there are limited data available on door-to-groin times (DGT) for EVT [8-10].

The Greater Amsterdam Area is a densely populated part of the Netherlands with generally well-organized stroke care and short treatment times for reperfusion therapy $[11,12]$. As a result, we hypothesized that presentation outside office hours would not lead to treatment delays for both IVT and EVT. 


\section{Methods}

\section{Study design}

We performed an observational cohort study in the Greater Amsterdam Area, which consists of 13 primary stroke centers (PSC), 1 general hospital, and one comprehensive stroke center (CSC), with a surface area of $2670 \mathrm{~km}^{2}$, and 2.76 million inhabitants. The participating hospitals are listed in Supplemental Table 1. All patients with AIS who received reperfusion therapy between January 2009 and December 2015 (IVT) and between April 2014 and December 2017 (EVT) were included.

\section{Patient selection}

Patients were identified from local prospective stroke registries. According to time of arrival at the Emergency Department (ED), we categorized patients as presenting during "office hours" or "outside office hours". Office hours were defined as Monday until Friday from 8 AM until 5 PM, with the exception of public holidays. If arrival time at ED was not recorded, we estimated the time by subtracting the median door-to-CT time from the time of the non-contrast CT. For patients receiving EVT, categorization was based on the time of presentation at the ED of the first hospital (i.e. for transfer patients the door time of the PSC was used). Because transfer patients have two door times (both door of PSC and CSC), we also categorized these patients based on the time of presentation at the ED of the CSC in a subgroup analysis. Based on the annual number of IVT procedures, hospitals were categorized as low-volume ( $\leq 24$ IVT treated patients per year), medium-volume (25-49) or high-volume ( $\geq 50$ ), as done previously [13]. Patients with an in-hospital stroke or for whom the time of presentation could not be ascertained were excluded.

\section{Data collection and outcomes}

We collected individual patient data extracted from medical records [12]. The institutional review board of the Amsterdam University Medical Centers, location AMC approved the study and waived the need for written informed consent from individual patients. Primary outcomes were the door-to-needle time (DNT) for patients receiving IVT and door-to-groin time (DGT) for patients receiving EVT. DGT was defined as the time interval between presentation at the first hospital and time of puncture of the groin. For patients that were transferred from a PSC towards the CSC to receive EVT, we used the door time of the PSC. Other outcomes were the modified Rankin Scale scores (mRS) after 3 months, in-hospital mortality, symptomatic intracranial hemorrhage (sICH, according to ECASS III criteria), infections (pneumonia and urinary tract), and Intensive Care Unit (ICU) admission.

\section{Statistical analysis}

We compared patients presenting outside office hours vs. during office hours. The results for IVT and EVT are reported separately and patients who received both IVT and EVT were analyzed in both groups. Baseline characteristics were compared using Chi-square test for categorical variables, independent samples $T$ test for normally distributed continuous variables, and Mann-Whitney $U$ test for nonnormally distributed continuous variables. Time intervals are expressed as medians with interquartile ranges (IQR). For regression analyses, missing data were imputed using multiple imputations by chained equations (MICE) based on relevant covariates and outcome.

We used multivariable linear regression analysis to evaluate the association between presentation outside office hours and door-to-treatment times. Presentation during office hours was used as the reference category. For DNT, we adjusted for the following pre-specified prognostic factors: age, sex, prior ischemic stroke/TIA, use of antithrombotic therapy, pre-stroke mRS, baseline NIHSS, systolic blood pressure, onset-to-door time, and hospital volume. For DGT, we used the same co-variables and additionally adjusted for treatment with IVT. We used multivariable binary logistic regression analysis to evaluate the association between presentation outside office hours and clinical and radiological outcomes, adjusting for the following pre-specified variables: age, sex, prior ischemic stroke/TIA, use of antithrombotic therapy, pre-stroke mRS, baseline NIHSS, onset-to-needle time, hospital volume, and treatment with IVT (for EVT patients only). Finally, we performed a secondary analysis of patients receiving EVT based on the time of presentation at the ED of the CSC. SPSS version 25 was used for all statistical analyses.

\section{Results}

In total, 4677 patients with AIS were treated with reperfusion therapy in the study period (Fig. 1). Of these, 433 were excluded because of in-hospital stroke $(n=177)$, unknown time of arrival ( $n=41)$, or unknown door-to-treatment time $(n=215)$. Therefore, data of 4244 patients were included in the analysis, of whom 4161 (98\%) received IVT and $395(9.3 \%)$ EVT. There were $311 / 395(78.7 \%)$ patients that received both IVT and EVT. In total, 2450/4161 (58.9\%) and 239/395 $(60.5 \%)$ patients treated with IVT and EVT, respectively, presented outside office hours. The number of EVT 
Fig. 1 Flowchart for patient selection. There were 311 patients that received both IVT and EVT

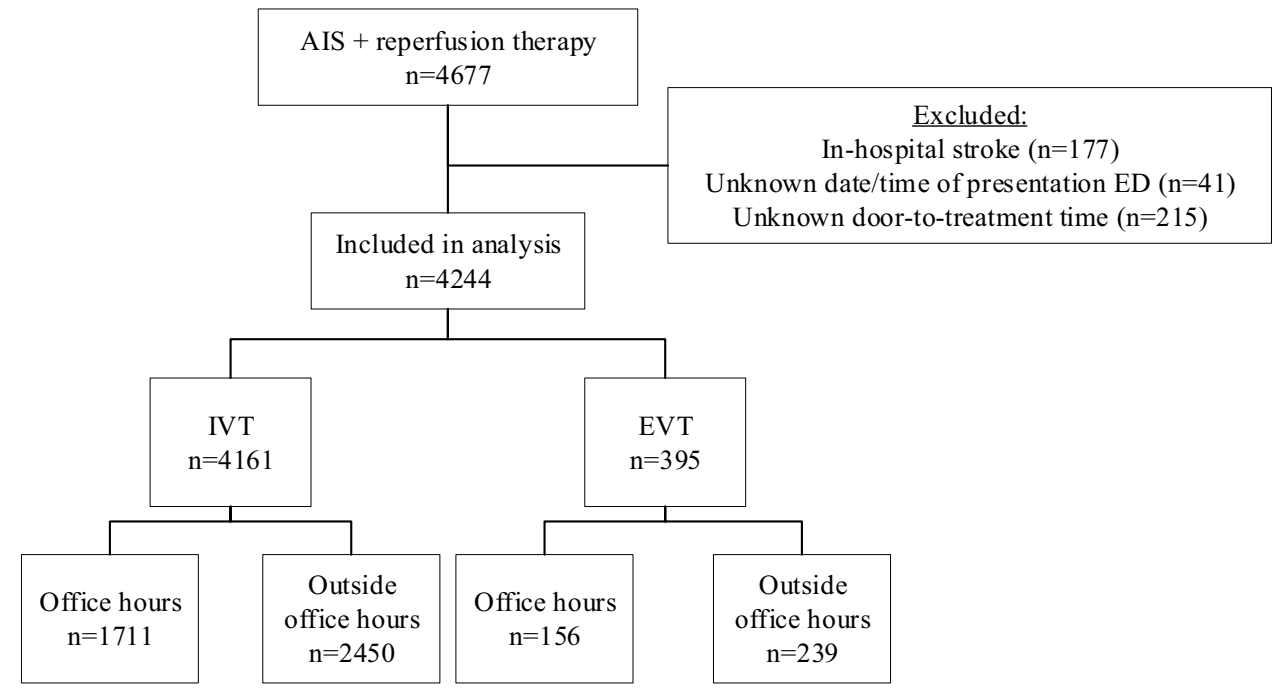

patients presented outside office hours increased per year (2014: 38.7\% , 2015: 59.2\%, 2016: 62.1\%, 2017: 64.5\%).

Intravenous thrombolysis (IVT) patients who presented outside office hours were more often men $(55.5 \%$ vs. $51.5 \%$, $p=0.010)$ and were slightly younger (70 vs. 71 years, $p=0.015$ ), compared to patients who presented during office hours (Table 1). For patients who received EVT, median onset-to-door time was longer for patients presented outside office hours (62 vs. $51 \mathrm{~min}, p=0.001$ ). There were no other statistically significant differences between the two groups.

\section{Outcomes IVT}

The median unadjusted DNT was slightly longer for patients presented outside office hours than for those who arrived during office hours ( 32 vs. $30 \mathrm{~min}, p=0.024$, Table 2 ) and this difference persisted after adjustment for potential confounders (adjusted difference $2.5 \mathrm{~min}, 95 \%$ CI 0.7-4.2). There were no differences regarding in-hospital mortality (7.4\% vs. $7.8 \%$, aOR $0.95,95 \%$ CI $0.73-1.23$ ), mRS 0-2 at 3 months (37.8\% vs. $36.2 \%$, aOR 1.02 , 95\% CI $0.87-1.20$ ), and frequency of sICH ( $4.2 \%$ vs. $4.8 \%$, aOR $0.86,95 \%$ CI 0.63-1.18). Patients presenting outside office hours did more often develop pneumonia ( $7.2 \%$ vs. $5.3 \%$, aOR $1.51,95 \%$ CI 1.13-2.00).

\section{Outcomes EVT}

There was no difference in DGT between patients presenting outside versus during office hours (both $130 \mathrm{~min}$, adjusted time difference $7.0 \mathrm{~min}, 95 \% \mathrm{CI}-4.2$ to 18.1 , Table 3 ). We also found no difference in DGT for transferred (144 vs. 147 min, adjusted time difference $7.9,95 \% \mathrm{CI}-5.2$ to 21.1 ) or directly (103 vs. $89 \mathrm{~min}$, adjusted time difference 5.6, $95 \%$ CI -12.7 to 23.8 ) presented patients. In-hospital mortality (outside office hours vs. during office hours: $11.7 \%$ vs. $16.0 \%$, aOR $1.02,95 \%$ CI $0.47-2.24)$, mRS $0-2$ at 3 months ( $38.8 \%$ vs. $39.7 \%$, aOR 0.92 , $95 \%$ CI $0.52-1.65)$, and sICH (9.2\% vs. $12.7 \%$, aOR $0.69,95 \%$ CI $0.30-1.57$ ) also did not differ between groups. Fourteen patients arrived at the CSC outside office hours, while initial presentation at PSC was during office hours. In addition, seven patients arrived at the CSC during office hours, while initial presentation at PSC was outside office hours. If we used the door time of the CSC instead of the PSC to categorize patients, there were also no statistically significant differences between the two groups regarding any of the outcomes (Supplemental Table 2).

\section{Discussion}

In this study, $60 \%$ of the patients treated with reperfusion therapy presented outside office hours. Presentation outside office hours was associated with a 2 min delay in DNT, which is unlikely to be clinically relevant. Presentation outside office hours was not associated with increased treatment times for patients undergoing EVT. There was also no difference in functional outcome or risk of sICH between patients presenting during versus outside office hours. This emphasizes our hypothesis that in a well-organized acute stroke care region, presentation outside office hours does not necessarily lead to worse outcomes.

A number of studies previously evaluated a possible "off hours" effect in patients receiving IVT [7,14-16]. The results of our study are generally in line with the results from a large European, multicenter cohort (TRISP), which also found a 2 min increase in DNT for presentation outside office hours. Though the increased DNT observed in TRISP was statistically significant, this study also found no association with clinical outcome, providing further 
Table 1 Baseline characteristics

\begin{tabular}{|c|c|c|c|c|c|c|}
\hline & \multicolumn{3}{|c|}{ Intravenous thrombolysis } & \multicolumn{3}{|l|}{ Endovascular treatment } \\
\hline & Office hours, $n=1711$ & $\begin{array}{l}\text { Outside office } \\
\text { hours, } n=2450\end{array}$ & $p$ value & Office hours, $n=156$ & $\begin{array}{l}\text { Outside office } \\
\text { hours, } n=239\end{array}$ & $p$ value \\
\hline Male sex- $n(\%)$ & $880 / 1709(51.5)$ & $1358 / 2446(55.5)$ & 0.010 & $88 / 156(56.4)$ & $123 / 239(51.5)$ & 0.335 \\
\hline Mean age in years $\pm \mathrm{SD}$ & $71 \pm 14$ & $70 \pm 14$ & 0.015 & $68 \pm 14$ & $67 \pm 14$ & 0.841 \\
\hline $\begin{array}{l}\text { Mean systolic blood pressure in } \\
\mathrm{mmHg} \pm \mathrm{SD}^{\mathrm{a}}\end{array}$ & $156 \pm 26$ & $155 \pm 25$ & 0.178 & $148 \pm 25$ & $145 \pm 23$ & 0.232 \\
\hline $\begin{array}{l}\text { Mean diastolic blood pressure in } \\
\mathrm{mmHg} \pm \mathrm{SD}^{\mathrm{b}}\end{array}$ & $85 \pm 16$ & $85 \pm 16$ & 0.560 & $83 \pm 17$ & $82 \pm 14$ & 0.516 \\
\hline Median NIHSS (IQR) ${ }^{\mathrm{d}}$ & $6(4-12)$ & $7(4-13)$ & 0.093 & $17(13-21)$ & $17(13-21)$ & 0.886 \\
\hline Pre-stroke $m R S \geq 2-n(\%)$ & 96/897 (10.7) & $121 / 1301(9.3)$ & 0.279 & $8 / 144(5.6)$ & $14 / 210(6.7)$ & 0.671 \\
\hline $\begin{array}{l}\text { Transfer from primary stroke center- } n \\
(\%)\end{array}$ & N/A & N/A & N/A & $104 / 156(66.7)$ & $162 / 239(67.8)$ & 0.817 \\
\hline \multicolumn{7}{|l|}{ Risk factors- $n(\%)$} \\
\hline Atrial fibrillation & $155 / 1690(9.2)$ & $221 / 2403(9.2)$ & 0.978 & 28/146 (19.2) & $47 / 212(22.2)$ & 0.494 \\
\hline Diabetes mellitus & $271 / 1693(16.0)$ & $383 / 2402(15.9)$ & 0.957 & 25/146 (17.1) & $30 / 212(14.2)$ & 0.443 \\
\hline Hypertension & $729 / 1688(43.2)$ & $1016 / 2401(42.3)$ & 0.579 & $64 / 141(45.4)$ & $90 / 209(43.1)$ & 0.667 \\
\hline Prior ischemic stroke/TIA & $448 / 1691(26.5)$ & $609 / 2399(25.4)$ & 0.426 & 27/146 (18.5) & $28 / 212(13.2)$ & 0.173 \\
\hline \multicolumn{7}{|l|}{ Medication use- $n(\%)$} \\
\hline Anticoagulation* & $37 / 968(3.8)$ & $38 / 1309(2.9)$ & 0.224 & 26/144 (18.1) & 43/208 (20.7) & 0.543 \\
\hline \multicolumn{7}{|l|}{ Process measures } \\
\hline General anesthesia- $n(\%)$ & N/A & N/A & N/A & $43 / 146(48.3)$ & $46 / 231(51.7)$ & 0.094 \\
\hline $\begin{array}{l}\text { Median admission duration in days } \\
(\text { IQR })^{\mathrm{c}}\end{array}$ & $4(2-8)$ & $4(2-8)$ & 0.958 & $2(1-5)$ & $2(1-5)$ & 0.680 \\
\hline $\begin{array}{l}\text { Median onset to door (first hospital) } \\
\text { time in minutes (IQR) }\end{array}$ & $70(45-115)$ & $67(45-112)$ & 0.544 & $51(28-74)$ & $62(44-102)$ & 0.001 \\
\hline
\end{tabular}

$S D$ standard deviation, NIHSS National Institutes of Health Stroke Scale, $m R S$ modified Rankin Scale

*Direct oral anticoagulation, vitamin K antagonist or heparin

Number of missing values: aIVT: 91 (5.3\%) vs. 136 (5.6\%), EVT: 8 (5.1\%) vs. 26 (10.9\%), bIVT: 91 (5.3\%) vs. 140 (5.7\%), EVT: 8 (5.1\%) vs. 28 $(11.7 \%),{ }^{\mathrm{c}}$ IVT: $29(1.7 \%)$ vs. $52(2.1 \%)$, EVT: $9(5.8 \%)$ vs. $26(10.9 \%),{ }^{\mathrm{d}}$ IVT: $30(1.8 \%)$ vs. 60 (2.4\%), EVT: 10 (6.4\%) vs. 28 (11.7\%), ${ }^{\mathrm{e}}$ IVT: 159 (9.3\%) vs. $238(9.7 \%)$, EVT: 7 (4.5\%) vs. $7(2.9 \%)$

proof that these small DNT differences are unlikely to be clinically relevant [7]. While various studies have examined the off-hours effect for IVT, data on this topic for EVT are scarce. Previous studies did report prolonged treatment times for patients with EVT presenting outside office hours, but none of these found an effect on functional outcome at 90 days $[8-10,17]$. One of those studies used data of the MRCLEAN Registry, reporting on results of all patients treated with EVT between March 2014 and June 2016 in the Netherlands, also including patients from the Greater Amsterdam Area [10]. We, however, report results of patients that received EVT over a wider time span (March 2014 through January 2018), and in contrast, we found no differences in treatment times or functional outcome, regardless of presentation time. Our region may differ from other regions in the Netherlands. Since 2016, all hospitals in our region are committed to a protocol containing requirements for each participating center to ensure optimal 24/7-stroke service (StrokeNet).
Implementation of such a protocol may have helped to ensure the timely start of EVT.

Patients that received IVT outside office hours more often suffered from pneumonia. There is at least one other study that also reported this observation [3]. Dysphagia is a common symptom after stroke, and protocols include dysphagia screening to reduce the frequency of aspiration and pneumonia nowadays [18]. While dysphagia screening is routinely done in accordance with national guidelines, such a screening might be delayed in cases who present outside office hours, due to unavailability of specialized staff such as speech therapists.

One of the strengths of our study was that we included all consecutive AIS patients in a large region in the Netherlands, with 15 participating hospitals including both PSCs and a CSC. The in-hospital logistics of these 15 hospitals were comparable, due to the earlier mentioned StrokeNet protocol. Another strength is that we had little missing data for door-to-treatment times. Several limitations of this study 
Table 2 Outcomes for patients treated with intravenous thrombolysis

\begin{tabular}{|c|c|c|c|c|}
\hline & Office hours, $n=1711$ & $\begin{array}{l}\text { Outside office hours, } \\
N=2450\end{array}$ & Unadjusted Beta/OR (95\% CI) & $\begin{array}{l}\text { Adjusted Beta*/ } \\
\text { OR**, }(95 \% \text { CI })\end{array}$ \\
\hline Median DNT (IQR) in minutes & $30(22-44)$ & $32(23-45)$ & $1.6(-0.2$ to 3.4$)$ & $2.5(0.7-4.2)$ \\
\hline $\mathrm{DNT}<60 \min -n(\%)$ & 1490/1711 (87.1) & $2090 / 2450(85.3)$ & $0.86(0.72$ to 1.03$)$ & $0.78(0.64-0.95)$ \\
\hline $\mathrm{DNT}<30 \mathrm{~min}-n(\%)$ & 778/1711 (45.5) & $1061 / 2450(43.3)$ & $0.92(0.81$ to 1.04$)$ & $0.86(0.75-0.98)$ \\
\hline Median ONT $(\mathrm{IQR})^{\mathrm{a}}$ in minutes & $107(78-160)$ & $108(80-160)$ & $2.5(-1.9$ to 6.8$)$ & $2.5(0.7-4.3)$ \\
\hline In-hospital mortality— $n(\%)$ & $133 / 1702(7.8)$ & $179 / 2435(7.4)$ & $0.94(0.74$ to 1.18$)$ & $0.95(0.73-1.23)$ \\
\hline Mortality after 3 months $-n(\%)$ & $175 / 1222(14.3)$ & 256/1761 (14.5) & $1.02(0.83$ to 1.25$)$ & $1.10(0.86-1.42)$ \\
\hline mRS $0-2$ after 3 months $-n(\%)$ & $519 / 1432(36.2)$ & $761 / 2014(37.8)$ & $1.07(0.93$ to 1.23$)$ & $1.02(0.87-1.20)$ \\
\hline Symptomatic ICH-n(\%) & $80 / 1661(4.8)$ & $100 / 2386(4.2)$ & $0.87(0.64$ to 1.17$)$ & $0.86(0.63-1.18)$ \\
\hline Pneumonia- $n(\%)$ & $90 / 1711(5.3)$ & $177 / 2450(7.2)$ & $1.40(1.08$ to 1.82$)$ & $1.51(1.13-2.00)$ \\
\hline Urinary tract infection- $n(\%)$ & 98/1711 (5.7) & $122 / 2450(5.0)$ & $0.86(0.66$ to 1.13$)$ & $0.90(0.67-1.20)$ \\
\hline ICU admission— $n(\%)$ & 65/1711 (3.8) & $102 / 2450(4.2)$ & $1.10(0.80$ to 1.51$)$ & $1.10(0.78-1.55)$ \\
\hline
\end{tabular}

$D N T$ door-to-needle time, $O N T$ onset-to-needle time, $O T D$ onset-to-door time, $I C H$ intracranial hemorrhage, $I C U$ intensive care unit

*Adjusted for age, sex, prior ischemic stroke/TIA, use of antithrombotic therapy, pre-stroke mRS, baseline NIHSS score, systolic blood pressure, onset-to-door time, hospital volume (for OTD as dependent variable excluding onset-to-door time)

**Adjusted for age, sex, prior ischemic stroke/TIA, use of antithrombotic therapy, pre-stroke mRS, baseline NIHSS, onset-to-needle time, and hospital volume

Number of missing values: ${ }^{\mathrm{a}} 148(8.6 \%)$ vs. $221(9.0 \%)$

Table 3 Outcomes for patients treated with endovascular treatment

\begin{tabular}{|c|c|c|c|c|}
\hline & Office hours, $n=156$ & $\begin{array}{l}\text { Outside office } \\
\text { hours, } N=239\end{array}$ & Unadjusted Beta/OR (95\% CI) & $\begin{array}{l}\text { Adjusted Beta*/ } \\
\text { OR }^{* *},(95 \% \text { CI })\end{array}$ \\
\hline Median DGT (IQR) in minutes & $130(96-170)$ & $130(107-175)$ & $6.2(-5.2$ to 17.6$)$ & $7.0(-4.2$ to 18.1$)$ \\
\hline Direct patients $(n=129)$ & $89(65-117)$ & $103(73-122)$ & $6.7(-11.1$ to 24.5$)$ & $5.6(-12.7$ to 23.8$)$ \\
\hline Transfer patients $(n=266)$ & $147(119-180)$ & $144(120-187)$ & $5.1(-7.5$ to 17.7$)$ & $7.9(-5.2$ to 21.1$)$ \\
\hline $\begin{array}{l}\text { Median DGT (door CSC) in min- } \\
\text { utes, only transfer patients (IQR) }\end{array}$ & $35(25-51)$ & $34(23-52)$ & $-2.1(-11.8$ to 7.7$)$ & $2.0(-8.0$ to 12.0$)$ \\
\hline $\begin{array}{l}\text { Median door-to-door time in min- } \\
\text { utes, only transfer patients (IQR) }\end{array}$ & $102(79-134)$ & $105(83-133)$ & $4.1(-7.7$ to 15.8$)$ & $5.6(-6.4$ to 17.7$)$ \\
\hline In-hospital mortality- $n(\%)$ & $25 / 156(16.0)$ & $28 / 239(11.7)$ & $0.70(0.39$ to 1.24$)$ & $1.02(0.47$ to 2.24$)$ \\
\hline Mortality after 3 months $-n(\%)$ & $39 / 134(29.1)$ & $61 / 207(29.5)$ & $1.02(0.63$ to 1.64$)$ & $1.09(0.55$ to 2.16$)$ \\
\hline mRS $0-2$ after 3 months $-n(\%)$ & $58 / 146(39.7)$ & $85 / 219(38.8)$ & $0.96(0.63$ to 1.48$)$ & $0.92(0.52$ to 1.65$)$ \\
\hline Direct patients $(n=129)$ & $24 / 48(50.0)$ & $31 / 70(44.3)$ & $0.80(0.38$ to 1.66$)$ & $0.70(0.23$ to 2.11$)$ \\
\hline Transfer patients $(n=266)$ & $34 / 98(34.7)$ & $54 / 149(36.2)$ & 1.07 (0.63 to 1.82$)$ & $1.01(0.48$ to 2.13$)$ \\
\hline Symptomatic ICH-n (\%) & $16 / 126(12.7)$ & $17 / 184(9.2)$ & $0.70(0.34$ to 1.44$)$ & $0.69(0.30$ to 1.57$)$ \\
\hline Pneumonia- $n(\%)$ & $17 / 156(10.9)$ & $25 / 239(10.5)$ & $0.96(0.50$ to 1.83$)$ & $1.77(0.73$ to 4.33$)$ \\
\hline Urinary tract infection- $n(\%)$ & $5 / 156(3.2)$ & $9 / 239(3.8)$ & $1.18(0.39$ to 3.59$)$ & $1.60(0.46$ to 5.53$)$ \\
\hline ICU admission- $n(\%)$ & $39 / 156(25.0)$ & $38 / 239(15.9)$ & $0.57(0.34$ to 0.94$)$ & $0.68(0.35$ to 1.33$)$ \\
\hline
\end{tabular}

$D G T$ door-to-groin time, $I Q R$ interquartile range, $S D$ standard deviation, $I C H$ intracranial hemorrhage, $I C U$ intensive care unit

*Adjusted for age, sex, prior ischemic stroke/TIA, use of antithrombotic therapy, pre-stroke mRS, baseline NIHSS score, systolic blood pressure, onset-to-needle time

${ }^{* *}$ Adjusted for age, sex, IVT treatment, prior ischemic stroke/TIA, use of antithrombotic therapy, pre-stroke mRS, baseline NIHSS, onset-toneedle time

should be noted. First, while patients were derived from local prospective stroke registries, a substantial proportion of the data were collected retrospectively. This explains the relatively high proportion of missing data on for example pre-stroke mRS. More importantly, mRS scores at 90 days were missing for $52 \%$ of patients treated with IVT, and only for $7.5 \%$ of patients treated with EVT. We tried to account for these missing values using multiple imputation. 
However, the results of functional outcome in IVT patients should be interpreted with caution, as these could be influenced by bias. Third, some patients, especially those transferred from a PSC to a CSC for EVT, initially presented during office hours, but underwent EVT outside office hours. However, the results of the secondary analysis based on the presentation time at the ED of the CSC do not seem to suggest that this led to a substantial distortion of the results. Fourth, even though data on door-to-treatment times were almost complete, we did not collect extra time points such as door-to-imaging times, and therefore, we cannot say anything about other delays that can appear outside office hours. Finally, we did not have data on patients that encountered significant transfer delays and as a result were ineligible for reperfusion therapy. Theoretically, this could differ between patients who presented during vs. outside office hours.

In conclusion, the results of our study suggest that in a well-organized acute stroke care region with overall low DNT and DGT, presentation outside office hours does not necessarily lead to a clinically relevant increase in treatment times for either IVT or EVT.

Acknowledgements We would like to thank Iris de Brouwer, medical student, for her help collecting data.

\section{Funding None.}

\section{Compliance with ethical standards}

\section{Conflicts of interest None.}

Open Access This article is licensed under a Creative Commons Attribution 4.0 International License, which permits use, sharing, adaptation, distribution and reproduction in any medium or format, as long as you give appropriate credit to the original author(s) and the source, provide a link to the Creative Commons licence, and indicate if changes were made. The images or other third party material in this article are included in the article's Creative Commons licence, unless indicated otherwise in a credit line to the material. If material is not included in the article's Creative Commons licence and your intended use is not permitted by statutory regulation or exceeds the permitted use, you will need to obtain permission directly from the copyright holder. To view a copy of this licence, visit http://creativecommons.org/licenses/by/4.0/.

\section{References}

1. Saver JL, Fonarow GC, Smith EE, Reeves MJ, Grau-sepulveda MV, Hernandez AF et al (2013) Time to treatment with intravenous tissue plasminogen activator and outcome from acute ischemic stroke. JAMA 309(23):2480-2488

2. Fransen PSS, Berkhemer OA, Lingsma HF, Beumer D, van den Berg LA, Yoo AJ et al (2015) Time to reperfusion and treatment effect for acute ischemic stroke: a randomized clinical trial. JAMA Neurol. 73:1-7

3. Reeves MJ, Smith E, Fonarow G, Hernandez A, Pan W, Schwamm LH (2009) Off-hour admission and in-hospital stroke case fatality in the get with the guidelines-stroke program. Stroke 40(2):569-576
4. Campbell JTP, Bray BD, Hoffman AM, Kavanagh SJ, Rudd AG, Tyrrell PJ (2014) The effect of out of hours presentation with acute stroke on processes of care and outcomes: analysis of data from the Stroke Improvement National Audit Programme (SINAP). PLoS ONE 9(2):1-7

5. Albright KC, Savitz SI, Raman R, Martin-Schild S, Broderick J, Ernstrom K et al (2012) Comprehensive stroke centers and the "Weekend Effect": the SPOTRIAS experience on behalf of the SPOTRIAS investigators. Cerebrovasc Dis 34(5-6):424-429

6. Jauss M, Schütz HJ, Tanislav C, Misselwitz B, Rosenow F (2010) Effect of daytime, weekday and year of admission on outcome in acute ischaemic stroke patients treated with thrombolytic therapy. Eur J Neurol 17(4):555-561

7. Zonneveld TP, Curtze S, Zinkstok SM, Gensicke H, Moulin S, Scheitz JF, Seiffge DJ, Hametner C, Heldner MR, Traenka C, Erdur H, Baharoglu I, Martinez-Majander N, Pezzini A, Zini A, Padjen V, Correia PN, Strbian D, Michel P, Béjot Y, Arnold M, Leys D, Ringl NPT collaborators (2017) Non-office-hours admission affects intravenous thrombolysis treatment times and clinical outcome. J Neurol Neurosurg Psychiatry. 89(9):1005-1007

8. Mpotsaris A, Kowoll A, Weber W, Kabbasch C, Weber A, Behme D (2015) Endovascular stroke therapy at nighttime and on weekends-as fast and effective as during normal business hours? J Vasc Interv Neurol 8(1):39-45

9. Almekhlafi MA, Hockley A, Desai JA, Nambiar V, Mishra S, Volny O et al (2014) Overcoming the evening/weekend effects on time delays and outcomes of endovascular stroke therapy: The Calgary Stroke Program experience. J Neurointerv Surg 6(10):729-732

10. Hinsenveld WH, de Ridder IR, van Oostenbrugge RJ, Vos JA, Groot AE, Coutinho JM et al (2019) Workflow intervals of endovascular acute stroke therapy during on- versus off-hours. Stroke 50(10):2842-2850

11. Jansen IGH, Mulder MJHL, Goldhoorn R-JB (2018) Endovascular treatment for acute ischaemic stroke in routine clinical practice: prospective, observational cohort study (MR CLEAN Registry). BMJ 360:k949

12. Groot AE, van Schaik IN, Visser MC, Nederkoorn PJ, Limburg M, Aramideh M et al (2016) Association between i.v. thrombolysis volume and door-to-needle times in acute ischemic stroke. J Neurol. 263(4):807-813

13. Bray BD, Campbell J, Geoffrey CC, Hoffman A, Tyrrell PJ, Wolfe CD et al (2013) Bigger, faster?: associations between hospital thrombolysis volume and speed of thrombolysis administration in acute ischemic stroke. Stroke 44:3129-3135

14. Fang K, Churilov L, Weir L, Dong Q, Davis S, Yan B (2014) Thrombolysis for acute ischemic stroke: do patients treated out of hours have a worse outcome? J Stroke Cerebrovasc Dis 23(3):427-432

15. Bodenant M, Leys D, Debette S, Cordonnier C, Dumont F, Hénon $\mathrm{H}$ et al (2010) Intravenous thrombolysis for acute cerebral ischaemia: comparison of outcomes between patients treated at working versus nonworking hours. Cerebrovasc Dis 30(2):148-156

16. Kim SK, Lee SY, Bae HJ, Lee YS, Kim SY, Kang MJ et al (2009) Pre-hospital notification reduced the door-to-needle time for iv t-PA in acute ischaemic stroke. Eur J Neurol 16(12):1331-1335

17. Nikoubashman O, Schürmann K, Othman AE, Bach JP, Wiesmann M, Reich A (2018) Improvement of endovascular stroke treatment: a 24-hour neuroradiological on-site service is not enough. Biomed Res Int 2018:9548743

18. Palli C, Fandler S, Doppelhofer K, Niederkorn K, Enzinger C, Vetta $C$ et al (2017) Early dysphagia screening by trained nurses reduces pneumonia rate in stroke patients: a clinical intervention study. Stroke 48(9):2583-2585 


\section{Affiliations}

\section{A. E. Groot ${ }^{1}$ - H. de Bruin ${ }^{1}$. T. T. M. Nguyen ${ }^{2}$ - M. Kappelhof ${ }^{3}$. F. de Beer ${ }^{4}$ - M. C. Visser ${ }^{5}$. C. P. Zwetsloot ${ }^{6}$. P. H. A. Halkes ${ }^{7}$. J. de Kruijk ${ }^{8}$. W. D. M. van der Meulen ${ }^{9}$. T. C. van der Ree ${ }^{10}$. V. I. H. Kwa ${ }^{11}$. S. M. van Schaik ${ }^{12}$. L. Hani ${ }^{13}$ - R. van den Berg $^{3}$ - M. E. S. Sprengers ${ }^{3}$ - S. D. Roosendaal ${ }^{3}$ - B. J. Emmer ${ }^{3}$. P. J. Nederkoorn ${ }^{1}$. C. B. L. M. Majoie ${ }^{3}$. Y. B. W. E. M. Roos ${ }^{1}$. J. M. Coutinho ${ }^{1}$}

$1 \quad$ Neurology, Amsterdam University Medical Center, University of Amsterdam, Meibergdreef 9, 1105 AZ Amsterdam, The Netherlands

2 Neurology, Leiden University Medical Center, Albinusdreef 2, 2333 ZA Leiden, The Netherlands

3 Radiology and Nuclear Medicine, Amsterdam University Medical Center, University of Amsterdam, Meibergdreef 9, 1105 AZ Amsterdam, The Netherlands

4 Neurology, Spaarne Gasthuis, Boerhaavelaan 22, 2035 RC Haarlem, The Netherlands

5 Neurology, Amsterdam University Medical Center, Vrije Universiteit Amsterdam, Boelelaan 1117-1118, 1081 HV Amsterdam, The Netherlands

6 Neurology, Dijklander, Waterlandlaan 250, 1441 RN Purmerend, The Netherlands
7 Neurology, Noord-West Ziekenhuisgroep, Wilhelminalaan 12, 1815 JD Alkmaar, The Netherlands

8 Neurology, Tergooi, Van Linschotenlaan 35, 1212 DR Hilversum, The Netherlands

9 Neurology, Rode Kruis, Vondellaan 13, 1942 LE Beverwijk, The Netherlands

10 Neurology, Dijklander, Maelsonstraat 3, 1624 NP Hoorn, The Netherlands

11 Neurology, OLVG-Oost, Oosterpark 9, 1091 AC Amsterdam, The Netherlands

12 Neurology, OLVG-West, Jan Tooropstraat 164, 1061 AE Amsterdam, The Netherlands

13 Neurology, Noord-West Ziekenhuisgroep, Huisduinerweg 3, 1782 GZ Den Helder, The Netherlands 